\title{
New Ring-Expansion Reactions of Hydroxy Propenoyl Cyclic Compounds Under the Palladium(0)/Phosphine-Catalyzed Conditions
}

\author{
Yoshimitsu Nagao, Satoru Tanaka, Akiharu Ueki, Masunori Kumazawa, Satoru Goto, \\ Takashi Ooi, Shigeki Sano, and Motoo Shiro
}

Supporting Information Available:

Typical experimental procedure for the synthesis of compounds 1b, c, 2ad, 3a-d, 4a, 5a, 6a-c, 7a, 9, 10, 14, and 15 and their physical and spectroscopic data.

(4 pages) 


\section{Experimental}

All melting points were determined on a Yanaco micro melting point apparatus and are uncorrected. IR spectra were obtained using a Perkin-Elmer 1720 or JASCO FT/IR-420 IR Fourier transform spectrophotometer. ${ }^{1} \mathrm{H}$ NMR $(300$ or $400 \mathrm{MHz})$ and ${ }^{13} \mathrm{C}$ NMR $(100 \mathrm{MHz})$ spectra were recorded on a JEOL JNM-AL300 or JEOL JNM-AL400 spectrometer. Chemical shifts are given in $\delta$ values (ppm) using tetramethylsilane (TMS) as an internal standard. HREI-MS were recorded on a JEOL JMS SX$102 \mathrm{~A}$ spectrometer. Elementary combustion analyses were performed using a Yanaco CHN CORDER MT-5. All reactions were monitored by TLC employing $0.25 \mathrm{~mm}$ silica gel plates (Merck 5715; 60 $\mathrm{F}_{254}$ ). Column chromatography was carried out on silica gel (Kanto Chemical N60 (spherical, neutral); 63-210 $\mu \mathrm{m}$ ). Anhydrous THF was used as purchased from Kanto Chemical. All other reagents were used as purchased.

General Procedure for Preparation of Indanones $1 \mathbf{b}$ and 1c. To a stirred solution of indanone (1.32 $\mathrm{g}, 10.0 \mathrm{mmol})$ in dry THF $(50 \mathrm{~mL})$ was added $\mathrm{NaH}(1.20 \mathrm{~g}, 60 \%$ in a mineral oil, $30.0 \mathrm{mmol})$ at $0{ }^{\circ} \mathrm{C}$ under argon. After being stirred for $10 \mathrm{~min}$, ethyl iodide $(2.40 \mathrm{~mL}, 30.0 \mathrm{mmol})$ was added and then the mixture was stirred at ambient temperature for $6 \mathrm{~h}$. The reaction mixture was cooled to $0{ }^{\circ} \mathrm{C}$, quenched with $1 \mathrm{~N} \mathrm{HCl}$, and extracted with ether. The ether extract was washed with an aqueous solution saturated with $\mathrm{Na}_{2} \mathrm{~S}_{2} \mathrm{O}_{3}$, water, brine, dried over $\mathrm{MgSO}_{4}$, and filtered. The filtrate was evaporated in vacuo to afford a crude product, which was purified by column chromatography on silica gel with $n$ hexane-AcOEt $(20: 1)$ to give 2,2-diethylindan-1-one (1b) $(1.63 \mathrm{~g}, 86 \%)$ as a pale yellow oil.

2,2-Diethylindan-1-one (1b). pale yellow oil; IR (neat) $1710 \mathrm{~cm}^{-1} ;{ }^{1} \mathrm{H}$ NMR $\left(400 \mathrm{MHz}, \mathrm{CDCl}_{3}\right) \delta 0.77$ $(\mathrm{t}, J=7.6 \mathrm{~Hz}, 6 \mathrm{H}), 1.57-1.74(\mathrm{~m}, 4 \mathrm{H}), 2.99(\mathrm{~s}, 2 \mathrm{H}), 7.35(\mathrm{t}, J=7.6 \mathrm{~Hz}, 1 \mathrm{H}), 7.45(\mathrm{~d}, J=7.6 \mathrm{~Hz}, 1 \mathrm{H})$, $7.58(\mathrm{t}, J=7.6 \mathrm{~Hz}, 1 \mathrm{H}), 7.73(\mathrm{~d}, J=7.6 \mathrm{~Hz}, 1 \mathrm{H})$; HREI-MS calcd for $\mathrm{C}_{13} \mathrm{H}_{16} \mathrm{O} \mathrm{MW} 188.1201$, found $m / z$ 188.1210 $\left(\mathrm{M}^{+}\right)$; Anal. Calcd for $\mathrm{C}_{13} \mathrm{H}_{16} \mathrm{O}: \mathrm{C}, 82.94 ; \mathrm{H}, 8.57$. Found: C, 82.70; H, 8.62.

2,2-Dibuthylindan-1-one (1c). yellowish oil; IR (neat) $1707 \mathrm{~cm}^{-1} ;{ }^{1} \mathrm{H} \mathrm{NMR}\left(400 \mathrm{MHz}, \mathrm{CDCl}_{3}\right) \delta 0.83$ $(\mathrm{t}, J=7.2 \mathrm{~Hz}, 6 \mathrm{H}), 0.98-1.31(\mathrm{~m}, 8 \mathrm{H}), 1.53-1.67(\mathrm{~m}, 4 \mathrm{H}), 3.00(\mathrm{~s}, 2 \mathrm{H}), 7.35(\mathrm{t}, J=7.6 \mathrm{~Hz}, 1 \mathrm{H}), 7.44(\mathrm{~d}$, $J=7.6 \mathrm{~Hz}, 1 \mathrm{H}), 7.58(\mathrm{t}, J=7.6 \mathrm{~Hz}, 1 \mathrm{H}), 7.73(\mathrm{~d}, J=7.6 \mathrm{~Hz}, 1 \mathrm{H}) ;$ HREI-MS calcd for $\mathrm{C}_{17} \mathrm{H}_{24} \mathrm{O}_{1} \mathrm{MW}$ 244.1827, found $m / z 244.1809\left(\mathrm{M}^{+}\right)$; Anal. Calcd for $\mathrm{C}_{17} \mathrm{H}_{24} \mathrm{O}_{1}$ : C, 83.55; H, 9.90. Found: C, 83.38; $\mathrm{H}$, 9.93.

General Procedure for Preparation of 1-Hydroxy-2,2-dialkyl-1-propenoylindan Derivatives 2a-d and 9. To a solution of methoxyallene $(1.05 \mathrm{~g}, 15.0 \mathrm{mmol})$ in dry THF $(15.0 \mathrm{~mL})$ was added $1.57 \mathrm{M} n$ butyllithium in $n$-hexane $(7.64 \mathrm{~mL}, 12.0 \mathrm{mmol})$ at $-78{ }^{\circ} \mathrm{C}$ under argon atmosphere, and the mixture was stirred at $-78^{\circ} \mathrm{C}$ for $30 \mathrm{~min}$. Then the mixture was added dropwise to a THF $(10.0 \mathrm{~mL})$ solution of 2,2dimethylindan-1-one (1a) $(1.60 \mathrm{~g}, 10.0 \mathrm{mmol})$ at $-78{ }^{\circ} \mathrm{C}$ in a cannula manner. After being stirred at -78 ${ }^{\circ} \mathrm{C}$ for $30 \mathrm{~min}$, the reaction mixture was acidified by $1 \mathrm{~N} \mathrm{HCl}$, homogenized with $\mathrm{MeOH}$ and then the reaction temperature was raised to room temperature. After being stirred at room temperature for $1 \mathrm{~h}$, the reaction mixture was extracted with ether. The ether extract was washed with brine, dried over $\mathrm{MgSO}_{4}$, and filtered. The filtrate was evaporated in vacuo to afford a crude product, which was purified by column chromatography on silica gel with $n$-hexane-AcOEt $(20: 1)$ to give 1-hydroxy-2,2-dimethyl1-propenoylindan (2a) (1.9 g, 88 \%) as a pale yellow oil.

1-Hydroxy-2,2-dimethyl-1-propenoylindan (2a). pale yellow oil; IR (neat) 3453, $1683 \mathrm{~cm}^{-1}$; ${ }^{1} \mathrm{H}$ NMR $\left(400 \mathrm{MHz}, \mathrm{CDCl}_{3}\right) \delta 1.05(\mathrm{~s}, 3 \mathrm{H}), 1.10(\mathrm{~s}, 3 \mathrm{H}), 2.92(\mathrm{~d}, J=16.1 \mathrm{~Hz}, 1 \mathrm{H}), 3.01(\mathrm{~d}, J=16.1 \mathrm{~Hz}, 1 \mathrm{H})$, $4.64(\mathrm{~s}, 1 \mathrm{H}), 5.62(\mathrm{dd}, J=10.4,1.6 \mathrm{~Hz}, 1 \mathrm{H}), 6.13(\mathrm{dd}, J=17.0,10.4 \mathrm{~Hz}, 1 \mathrm{H}), 6.43(\mathrm{dd}, J=17.0,1.6 \mathrm{~Hz}$, $1 \mathrm{H}), 7.16(\mathrm{~d}, J=7.6 \mathrm{~Hz}, 1 \mathrm{H}), 7.21-7.33(\mathrm{~m}, 3 \mathrm{H})$; HREI-MS calcd for $\mathrm{C}_{14} \mathrm{H}_{16} \mathrm{O}_{2}$ MW 216.1150, found $m / z 216.1162\left(\mathrm{M}^{+}\right)$; Anal. Calcd for $\mathrm{C}_{14} \mathrm{H}_{16} \mathrm{O}_{2}$ : C, 77.75; H, 7.46. Found: C, 77.48; H, 7.39. 
2,2-Diethyl-1-hydroxy-1-propenoylindan (2b). pale yellow oil; IR (neat) $3451,1681 \mathrm{~cm}^{-1}$; ${ }^{1} \mathrm{H}$ NMR $\left(400 \mathrm{MHz} \mathrm{CDCl}_{3}\right) \delta 0.75(\mathrm{t}, J=7.5 \mathrm{~Hz}, 3 \mathrm{H}), 0.84(\mathrm{t}, J=7.5 \mathrm{~Hz}, 3 \mathrm{H}), 1.40-1.59(\mathrm{~m}, 3 \mathrm{H}), 1.78-1.87(\mathrm{~m}$, $1 \mathrm{H}), 2.84(\mathrm{~d}, J=16.4 \mathrm{~Hz}, 1 \mathrm{H}), 3.06(\mathrm{~d}, J=16.4 \mathrm{~Hz}, 1 \mathrm{H}), 4.67(\mathrm{~s}, 1 \mathrm{H}), 5.60(\mathrm{dd}, J=10.3,1.7 \mathrm{~Hz}, 1 \mathrm{H})$, $6.13(\mathrm{dd}, J=17.1,10.3 \mathrm{~Hz}, 1 \mathrm{H}), 6.43(\mathrm{dd}, J=17.1,1.7 \mathrm{~Hz}, 1 \mathrm{H}), 7.09(\mathrm{~d}, J=7.6 \mathrm{~Hz}, 1 \mathrm{H}), 7.19-7.30(\mathrm{~m}$, $3 \mathrm{H}$ ); HREI-MS calcd for $\mathrm{C}_{16} \mathrm{H}_{20} \mathrm{O}_{2} \mathrm{MW} 244.1463$, found $m / z 244.1478\left(\mathrm{M}^{+}\right)$; Anal. Calcd for $\mathrm{C}_{16} \mathrm{H}_{20} \mathrm{O}_{2}$ : C, 78.65; H, 8.25. Found: C, 78.42; H, 8.24.

2,2-Dibutyl-1-hydroxy-1-propenoylindan (2c). yellowish oil; IR (neat) 3451, $1683 \mathrm{~cm}^{-1}$; ${ }^{1} \mathrm{H}$ NMR $\left(400 \mathrm{MHz}, \mathrm{CDCl}_{3}\right) \delta 0.84(\mathrm{q}, J=7.3 \mathrm{~Hz}, 6 \mathrm{H}), 1.07-1.48(\mathrm{~m}, 11 \mathrm{H}), 1.72-1.80(\mathrm{~m}, 1 \mathrm{H}), 2.84(\mathrm{~d}, J=16.4$ $\mathrm{Hz}, 1 \mathrm{H}), 3.07(\mathrm{~d}, J=16.4 \mathrm{~Hz}, 1 \mathrm{H}), 4.67(\mathrm{~s}, 1 \mathrm{H}), 5.59(\mathrm{dd}, J=10.3,1.7 \mathrm{~Hz}, 1 \mathrm{H}), 6.13(\mathrm{dd}, J=16.9,10.3$ $\mathrm{Hz}, 1 \mathrm{H}), 6.42(\mathrm{dd}, J=16.9,1.7 \mathrm{~Hz}, 1 \mathrm{H}), 7.09(\mathrm{~d}, J=7.3 \mathrm{~Hz}, 1 \mathrm{H}), 7.19-7.30(\mathrm{~m}, 3 \mathrm{H})$; HREI-MS calcd for $\mathrm{C}_{20} \mathrm{H}_{28} \mathrm{O}_{2}$ MW 300.2089, found $m / z 300.2118\left(\mathrm{M}^{+}\right)$; Anal. Calcd for $\mathrm{C}_{20} \mathrm{H}_{28} \mathrm{O}_{2}$ : C, 79.96; H, 9.39. Found: C, 79.72; H, 9.43.

1-Hydroxy-1-propenoylindan-2-spiro-1'-cyclopentane (2d). colorless oil; IR (neat) $3583,1684 \mathrm{~cm}^{-1}$; ${ }^{1} \mathrm{H}$ NMR $\left(400 \mathrm{MHz}, \mathrm{CDCl}_{3}\right) \delta 1.34-1.53(\mathrm{~m}, 3 \mathrm{H}), 1.61-1.73(\mathrm{~m}, 4 \mathrm{H}), 2.08-2.15(\mathrm{~m}, 1 \mathrm{H}), 2.93(\mathrm{~d}, J=$ $16.0 \mathrm{~Hz}, 1 \mathrm{H}), 3.04(\mathrm{~d}, J=16.0 \mathrm{~Hz}, 1 \mathrm{H}), 4.72(\mathrm{~s}, 1 \mathrm{H}), 5.62(\mathrm{dd}, J=10.4,1.7 \mathrm{~Hz}, 1 \mathrm{H}), 6.16(\mathrm{dd}, J=17.1$, $10.4 \mathrm{~Hz}, 1 \mathrm{H}), 6.42(\mathrm{dd}, J=17.1,1.7 \mathrm{~Hz}, 1 \mathrm{H}), 7.16(\mathrm{~d}, J=7.3 \mathrm{~Hz}, 1 \mathrm{H}), 7.21-7.31$ (m, 3H); HREI-MS calcd for $\mathrm{C}_{16} \mathrm{H}_{18} \mathrm{O}_{2} \mathrm{MW} 242.1307$, found $\mathrm{m} / \mathrm{z} 242.1320\left(\mathrm{M}^{+}\right)$.

17-Hydroxy-3-methoxy-13-methyl-17-propenoyl-7,8,9,11,12,13,14,15,16,17-decahydro-6Hcyclopenta $[\boldsymbol{a}]$ phenanthren $(9)$. pale yellow powder; IR $(\mathrm{KBr}) 3492,1726,1682 \mathrm{~cm}^{-1}$; ${ }^{1} \mathrm{H}$ NMR $(400$ $\left.\mathrm{MHz}, \mathrm{CDCl}_{3}\right) \delta 0.99(\mathrm{~s}, 3 \mathrm{H}), 1.26-1.80(\mathrm{~m}, 8 \mathrm{H}), 1.88-1.95(\mathrm{~m}, 2 \mathrm{H}), 2.07-2.14(\mathrm{~m}, 1 \mathrm{H}), 2.22-2.27(\mathrm{~m}$, $1 \mathrm{H}), 2.37-2.45(\mathrm{~m}, 1 \mathrm{H}), 2.84-2.88(\mathrm{~m}, 3 \mathrm{H}), 3.77(\mathrm{~s}, 3 \mathrm{H}), 5.73(\mathrm{dd}, \mathrm{J}=10.5,2.0 \mathrm{~Hz}, 1 \mathrm{H}), 6.40(\mathrm{dd}, \mathrm{J}=$ 17.1, 2.0 Hz, 1H), $6.62(\mathrm{~d}, \mathrm{~J}=2.7 \mathrm{~Hz}, 1 \mathrm{H}), 6.69(\mathrm{dd}, \mathrm{J}=8.3,2.7 \mathrm{~Hz}, 1 \mathrm{H}), 6.96(\mathrm{dd}, \mathrm{J}=17.1,10.5 \mathrm{~Hz}$, $1 \mathrm{H}), 7.14(\mathrm{~d}, \mathrm{~J}=8.3 \mathrm{~Hz}, 1 \mathrm{H})$; HREI-MS calcd for $\mathrm{C}_{12} \mathrm{H}_{28} \mathrm{O}_{3} \mathrm{MW} 340.2038$, found $\mathrm{m} / z$ 340.2041 $\left(\mathrm{M}^{+}\right)$.

Typical Procedure for the Ring Expansion Reaction. A mixture of 1-hydroxy-2,2-dimethyl-1propenoylindan (2a) $(31.8 \mathrm{mg}, 0.147 \mathrm{mmol}), \mathrm{Pd}\left(\mathrm{PPh}_{3}\right)_{4}(8.5 \mathrm{mg}, 0.007 \mathrm{mmol}), \mathrm{P}(o \text {-tolyl })_{3}(3.9 \mathrm{mg}$, $0.015 \mathrm{mmol})$ in THF $(1.5 \mathrm{~mL})$ was refluxed under argon atmosphere for $7 \mathrm{~h}$. The reaction mixture was evaporated in vacuo to afford a crude product, which was purified by column chromatography on silica gel with $n$-hexane-AcOEt $(10: 1)$ to give 2-hydroxy-3,3-dimethyl-2-vinyl-1-tetralone (3a) (29.3 mg, 92\%) as white crystals.

2-Hydroxy-3,3-dimethyl-2-vinyl-1-tetralone (3a). white crystals; mp 48.5-49.0 ${ }^{\circ} \mathrm{C}$ ( $n$-hexane); IR (KBr) 3469, $1685 \mathrm{~cm}^{-1}$; ${ }^{1} \mathrm{H}$ NMR $\left(300 \mathrm{MHz}, \mathrm{CDCl}_{3}\right) \delta 0.97(\mathrm{~s}, 3 \mathrm{H}), .1 .14(\mathrm{~s}, 3 \mathrm{H}), 2.74(\mathrm{~d}, J=17.6 \mathrm{~Hz}$, $1 \mathrm{H}), 3.17(\mathrm{~d}, J=17.6 \mathrm{~Hz}, 1 \mathrm{H}), 3.89(\mathrm{~s}, 1 \mathrm{H}), 5.24(\mathrm{dd}, J=10.7,1.5 \mathrm{~Hz}, 1 \mathrm{H}), 5.54(\mathrm{dd}, J=16.9,1.5 \mathrm{~Hz}$, $1 \mathrm{H}), 6.01(\mathrm{dd}, J=16.9,10.7 \mathrm{~Hz}, 1 \mathrm{H}), 7.24(\mathrm{~d}, J=7.7 \mathrm{~Hz}, 1 \mathrm{H}), 7.33(\mathrm{t}, J=7.7 \mathrm{~Hz}, 1 \mathrm{H}), 7.54(\mathrm{t}, J=7.7$ $\mathrm{Hz}, 1 \mathrm{H}), 7.98(\mathrm{~d}, J=7.7 \mathrm{~Hz}, 1 \mathrm{H})$; HREI-MS calcd for $\mathrm{C}_{14} \mathrm{H}_{16} \mathrm{O}_{2} \mathrm{MW} 216.1150$, found $\mathrm{m} / z 216.1166$ $\left(\mathrm{M}^{+}\right)$; Anal. Calcd for $\mathrm{C}_{14} \mathrm{H}_{16} \mathrm{O}_{2}$ : C, 77.75; H, 7.46. Found: C, 77.55; H, 7.49.

3,3-Diethyl-2-hydroxy-2-vinyl-1-tetralone (3b). pale yellow oil; IR (neat) $3470,1685 \mathrm{~cm}^{-1}$; ${ }^{1} \mathrm{H}$ NMR $\left(400 \mathrm{MHz} \mathrm{CDCl}_{3}\right) \delta 0.76(\mathrm{t}, J=7.4 \mathrm{~Hz}, 3 \mathrm{H}), 0.99(\mathrm{t}, J=7.4 \mathrm{~Hz}, 3 \mathrm{H}), 1.12(\mathrm{sext}, J=7.4 \mathrm{~Hz}, 1 \mathrm{H}), 1.42-$ $1.51(\mathrm{~m}, 1 \mathrm{H}), 1.69-1.87(\mathrm{~m}, 2 \mathrm{H}), 2.88(\mathrm{~d}, J=18.1 \mathrm{~Hz}, 1 \mathrm{H}), 2.93(\mathrm{~d}, J=18.1 \mathrm{~Hz}, 1 \mathrm{H}), 3.95(\mathrm{~s}, 1 \mathrm{H}), 5.24$ $(\mathrm{dd}, J=10.5,1.7 \mathrm{~Hz}, 1 \mathrm{H}), 5.59(\mathrm{dd}, J=16.6,1.7 \mathrm{~Hz}, 1 \mathrm{H}), 6.02(\mathrm{dd}, J=16.6,10.5 \mathrm{~Hz}, 1 \mathrm{H}), 7.25(\mathrm{~d}, J=$ $7.6 \mathrm{~Hz}, 1 \mathrm{H}), 7.33(\mathrm{t}, J=7.6 \mathrm{~Hz}, 1 \mathrm{H}), 7.54(\mathrm{td}, J=7.6,1.2 \mathrm{~Hz}, 1 \mathrm{H}), 7.97(\mathrm{dd}, J=7.6,1.2 \mathrm{~Hz}, 1 \mathrm{H})$; HREI-MS calcd for $\mathrm{C}_{16} \mathrm{H}_{20} \mathrm{O}_{2}$ MW 244.1463, found $\mathrm{m} / z$ 244.1468 (M ${ }^{+}$); Anal. Calcd for $\mathrm{C}_{16} \mathrm{H}_{20} \mathrm{O}_{2}: \mathrm{C}_{\text {, }}$ 78.65; H, 8.25. Found: C, 78.87; H, 8.32.

3,3-Dibutyl-2-hydroxy-2-vinyl-1-tetralone (3c). colorless oil; IR (neat) $3470,1685 \mathrm{~cm}^{-1}$; ${ }^{1} \mathrm{H}$ NMR $\left(400 \mathrm{MHz}, \mathrm{CDCl}_{3}\right) \delta 0.76-1.75(\mathrm{~m}, 18 \mathrm{H}), 2.90(\mathrm{~d}, J=17.8 \mathrm{~Hz}, 1 \mathrm{H}), 2.95(\mathrm{~d}, J=17.8 \mathrm{~Hz}, 1 \mathrm{H}), 3.96(\mathrm{~s}$, $1 \mathrm{H}), 5.23(\mathrm{~d}, J=10.5 \mathrm{~Hz}, 1 \mathrm{H}), 5.57(\mathrm{~d}, J=16.8 \mathrm{~Hz}, 1 \mathrm{H}), 6.01(\mathrm{dd}, J=10.5,16.8 \mathrm{~Hz}, 1 \mathrm{H}), 7.23-7.35$ $(\mathrm{m}, 1 \mathrm{H}), 7.34(\mathrm{t}, J=7.6 \mathrm{~Hz}, 1 \mathrm{H}), 7.54(\mathrm{t}, J=7.6 \mathrm{~Hz}, 1 \mathrm{H}), 7.97(\mathrm{~d}, J=7.6 \mathrm{~Hz}, 1 \mathrm{H})$; HREI-MS calcd for $\mathrm{C}_{20} \mathrm{H}_{28} \mathrm{O}_{2} \mathrm{MW} 300.2089$, found $\mathrm{m} / z 300.2095\left(\mathrm{M}^{+}\right)$; Anal. Calcd for $\mathrm{C}_{20} \mathrm{H}_{28} \mathrm{O}_{2}$ : C, 79.96; $\mathrm{H}, 9.39$. Found: C, 80.04; H, 9.50. 
2-Hydroxy-3-spiro-1'-cyclopentane-2-vinyl-1-tetralone (3d). colorless oil; IR (neat) $3481,1709 \mathrm{~cm}^{-1}$; ${ }^{1} \mathrm{H}$ NMR $\left(400 \mathrm{MHz}, \mathrm{CDCl}_{3}\right) \delta 1.14-1.18(\mathrm{~m}, 1 \mathrm{H}), 1.35-1.41(\mathrm{~m}, 1 \mathrm{H}), 1.56-1.73(\mathrm{~m}, 5 \mathrm{H}), 2.05-2.12(\mathrm{~m}$, S-31H), $2.90(\mathrm{~d}, J=17.6 \mathrm{~Hz}, 1 \mathrm{H}), 3.14(\mathrm{~d}, J=17.6 \mathrm{~Hz}, 1 \mathrm{H}), 3.93(\mathrm{~s}, 1 \mathrm{H}), 5.19$ (dd, $J=10.7,1.5 \mathrm{~Hz}$, $1 \mathrm{H}), 5.46(\mathrm{dd}, J=17.1,1.5 \mathrm{~Hz}, 1 \mathrm{H}), 6.08(\mathrm{dd}, J=17.1,10.5 \mathrm{~Hz}, 1 \mathrm{H}), 7.24(\mathrm{~d}, J=7.6 \mathrm{~Hz}, 1 \mathrm{H}), 7.34(\mathrm{t}, J$ $=7.6 \mathrm{~Hz}, 1 \mathrm{H}), 7.52(\mathrm{td}, J=7.6,1.2 \mathrm{~Hz}, 1 \mathrm{H}), 7.99(\mathrm{dd}, J=7.6,1.2 \mathrm{~Hz}, 1 \mathrm{H})$; HREI-MS calcd for $\mathrm{C}_{16} \mathrm{H}_{18} \mathrm{O}_{2}$ MW 242.1307, found $m / z 242.1324\left(\mathrm{M}^{+}\right)$; Anal. Calcd for $\mathrm{C}_{16} \mathrm{H}_{18} \mathrm{O}_{2}$ : C, 79.31; $\mathrm{H}, 7.49$. Found: C, 79.27; H, 7.60.

2-Ethyl-2-hydroxy-3,3-dimethyl-1-tetralone (7a). colorless needles.; mp 70.0-70.5 ${ }^{\circ} \mathrm{C}\left(\mathrm{CHCl}_{3}\right.$ - $n$ hexane-Et ${ }_{2} \mathrm{O}$ ); IR (KBr) 3476, $1678 \mathrm{~cm}^{-1} ;{ }^{1} \mathrm{H} \mathrm{NMR}\left(400 \mathrm{MHz}, \mathrm{CDCl}_{3}\right) \delta 0.70(\mathrm{t}, J=7.3 \mathrm{~Hz}, 3 \mathrm{H}), 0.93$ $(\mathrm{s}, 3 \mathrm{H}), 1.21(\mathrm{~s}, 3 \mathrm{H}), 1.65-1.74(\mathrm{~m}, 1 \mathrm{H}), 1.82-1.91(\mathrm{~m}, 1 \mathrm{H}), 2.74(\mathrm{~d}, J=17.7 \mathrm{~Hz}, 1 \mathrm{H}), 3.18(\mathrm{~d}, J=17.7$ $\mathrm{Hz}, 1 \mathrm{H}), 3.88(\mathrm{~s}, 1 \mathrm{H}), 7.21(\mathrm{~d}, J=7.6 \mathrm{~Hz}, 1 \mathrm{H}), 7.33(\mathrm{t}, J=7.6 \mathrm{~Hz}, 1 \mathrm{H}), 7.52(\mathrm{td}, J=7.6,1.2 \mathrm{~Hz}, 1 \mathrm{H})$, 7.95 (dd, $J=7.6,1.2 \mathrm{~Hz}, 1 \mathrm{H}$ ); HREI-MS calcd for $\mathrm{C}_{14} \mathrm{H}_{18} \mathrm{O}_{2} \mathrm{MW} 218.1307$, found $\mathrm{m} / z 218.1305\left(\mathrm{M}^{+}\right)$; Anal. Calcd for $\mathrm{C}_{14} \mathrm{H}_{18} \mathrm{O}_{2}$ : C, 77.03; H, 8.31. Found: C, 76.76; H, 8.28.

17 $\beta$-Hydroxy-3-methoxy-17 $\alpha$-vinyl- $D$-homoestra-1,3,5(10)-trien-17a-one (10). colorless plates; mp $214-215{ }^{\circ} \mathrm{C}\left(\mathrm{CHCl}_{3}-n\right.$-hexane-Et $\left.2 \mathrm{O}\right)$; IR $(\mathrm{KBr}) 3489,1712 \mathrm{~cm}^{-1} ;{ }^{1} \mathrm{H}$ NMR $\left(400 \mathrm{MHz}, \mathrm{CDCl}_{3}\right) \delta 0.82(\mathrm{~s}$, $3 \mathrm{H}), 1.34-1.60(\mathrm{~m}, 4 \mathrm{H}), 1.68-1.73(\mathrm{~m}, 2 \mathrm{H}), 1.89-1.95(\mathrm{~m}, 1 \mathrm{H}), 2.08-2.14(\mathrm{~m}, 1 \mathrm{H}), 2.26-2.34(\mathrm{~m}, 3 \mathrm{H})$, 2.51-2.67 (m, 2H), 2.83-2.88 (m, 2H), $3.78(\mathrm{~s}, 3 \mathrm{H}), 3.95(\mathrm{~s}, 1 \mathrm{H}), 5.33(\mathrm{dd}, J=10.5,1.8 \mathrm{~Hz}, 1 \mathrm{H}), 5.60$ $(\mathrm{dd}, J=16.6,1.8 \mathrm{~Hz}, 1 \mathrm{H}), 6.38(\mathrm{dd}, J=16.6,10.5 \mathrm{~Hz}, 1 \mathrm{H}), 6.63(\mathrm{~d}, J=2.8 \mathrm{~Hz}, 1 \mathrm{H}), 6.72(\mathrm{dd}, J=8.7$, $2.8 \mathrm{~Hz}, 1 \mathrm{H}), 7.21(\mathrm{~d}, J=8.7 \mathrm{~Hz}, 1 \mathrm{H})$; HREI-MS calcd for $\mathrm{C}_{22} \mathrm{H}_{28} \mathrm{O}_{3}$ MW 340.2039, found $m / z 340.2020$ $\left(\mathrm{M}^{+}\right)$; Anal. Calcd for $\mathrm{C}_{22} \mathrm{H}_{28} \mathrm{O}_{3}$ : C, 77.61; H, 8.29. Found: C, 77.46; H, 8.34.

17 $\alpha$-Ethyl-17 $\beta$-hydroxy- $D$-homoestra-1,3,5(10)-trien-17a-one (15). colorless plates; mp 150 - $151{ }^{\circ} \mathrm{C}$ $\left(\mathrm{CHCl}_{3}\right.$-n-hexane-Et $\left.2 \mathrm{O}\right)$; IR $(\mathrm{KBr}) 3488,1709 \mathrm{~cm}^{-1} ;{ }^{1} \mathrm{H} \mathrm{NMR}\left(400 \mathrm{MHz}, \mathrm{CDCl}_{3}\right) \delta 0.73(\mathrm{t}, J=7.3 \mathrm{~Hz}$, $3 \mathrm{H}), 0.77(\mathrm{~s}, 3 \mathrm{H}), 1.26-1.52(\mathrm{~m}, 4 \mathrm{H}), 1.74-1.92(\mathrm{~m}, 4 \mathrm{H}), 2.00-2.11(\mathrm{~m}, 2 \mathrm{H}), 2.21-2.53(\mathrm{~m}, 5 \mathrm{H}), 2.85-$ $2.87(\mathrm{~m}, 2 \mathrm{H}), 3.78(\mathrm{~s}, 3 \mathrm{H}), 3.82(\mathrm{~s}, 1 \mathrm{H}), 6.63(\mathrm{~d}, J=2.4 \mathrm{~Hz}, 1 \mathrm{H}), 6.72(\mathrm{dd}, J=8.6,2.4 \mathrm{~Hz}, 1 \mathrm{H}), 7.22(\mathrm{~d}$,

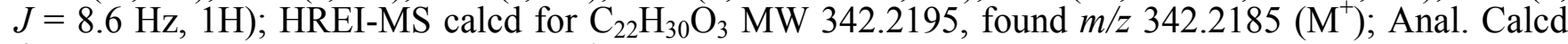
for $\mathrm{C}_{22} \mathrm{H}_{30} \mathrm{O}_{3}$ : C, 77.16; $\mathrm{H}, 8.83$. Found: C, 77.01; H, 8.60.

General Procedure for Preparation of Cyclic Hydroxy Conjugated Enones 4a and 5a. To a solution of methoxyallene $(105.1 \mathrm{mg}, 1.5 \mathrm{mmol})$ in dry THF $(1.5 \mathrm{~mL})$ was added $1.58 \mathrm{M} n$-butyllithium in $n$ hexane $(0.76 \mathrm{~mL}, 1.2 \mathrm{mmol})$ at $-78^{\circ} \mathrm{C}$ under argon atmosphere, and the mixture was stirred at $-78^{\circ} \mathrm{C}$ for $30 \mathrm{~min}$. Then the mixture was added dropwise to a THF $(1.0 \mathrm{~mL})$ solution of 2,2-dimethyl-1-tetralone $(174.2 \mathrm{mg}, 1.0 \mathrm{mmol})$ at $-78{ }^{\circ} \mathrm{C}$ in a cannula manner. After being stirred at $-78{ }^{\circ} \mathrm{C}$ for $1 \mathrm{~h}$, the reaction mixture was acidified by $1 \mathrm{~N} \mathrm{HCl}$, and then the reaction temperature was raised to room temperature. After being stirred for $16 \mathrm{~h}$, the reaction mixture was extracted with ether, and the ether extract was washed with brine, dried over $\mathrm{MgSO}_{4}$, and filtered. The filtrate was evaporated in vacuo to afford a crude product, which was purified by column chromatography on silica gel with $n$-hexane-AcOEt (20 : 1) to give 1-hydroxy-2,2-dimethyl-1-propenoyltetralin (4a) (136.5 mg, 59\%) as a colorless oil.

1-Hydroxy-2,2-dimethyl-1-propenoyltetralin (4a). colorless oil; IR (neat) 3454, $1681 \mathrm{~cm}^{-1}$; ${ }^{1} \mathrm{H}$ NMR $\left(400 \mathrm{MHz}, \mathrm{CDCl}_{3}\right) \delta 0.90(\mathrm{~s}, 3 \mathrm{H}), 1.05(\mathrm{~s}, 3 \mathrm{H}), 1.75-1.81(\mathrm{~m}, 1 \mathrm{H}), 1.95-2.03(\mathrm{~m}, 1 \mathrm{H}), 2.88-2.95(\mathrm{~m}$, $1 \mathrm{H}), 3.02-3.10(\mathrm{~m}, 1 \mathrm{H}), 4.73(\mathrm{~s}, 1 \mathrm{H}), 5.59(\mathrm{t}, J=6.1 \mathrm{~Hz}, 1 \mathrm{H}), 6.34(\mathrm{~d}, J=6.1 \mathrm{~Hz}, 2 \mathrm{H}), 7.14-7.24(\mathrm{~m}$, $4 \mathrm{H}) ;{ }^{13} \mathrm{C}-\mathrm{NMR}\left(75 \mathrm{MHz}, \mathrm{CDCl}_{3}\right) \delta 23.5,24.6,25.5,33.8,36.9,83.1,126.6,127.9,128.1,129.1,129.2$, 132.0, 136.3, 136.6, 201.4; HREI-MS calcd for $\mathrm{C}_{15} \mathrm{H}_{18} \mathrm{O}_{2} \mathrm{MW} 230.1307$, found $\mathrm{m} / \mathrm{z} 230.1319\left(\mathrm{M}^{+}\right)$.

1-Hydroxy-2,2-5-dimethy-1-propenoylbenzosuberan (5a). colorless powder; $\mathrm{mp} \quad 46.5-47.5{ }^{\circ} \mathrm{C}$ $\left(\mathrm{CHCl}_{3}-n\right.$-hexane-Et $\left.2 \mathrm{O}\right)$; IR (KBr) $3477,1698 \mathrm{~cm}^{-1} ;{ }^{1} \mathrm{H}$ NMR $\left(400 \mathrm{MHz}, \mathrm{CDCl}_{3}\right) \delta 0.72(\mathrm{~s}, 3 \mathrm{H}), 1.23(\mathrm{~s}$, $3 \mathrm{H}), 1.55-1.63(\mathrm{~m}, 1 \mathrm{H}), 1.73-1.82(\mathrm{~m}, 2 \mathrm{H}), 2.44-2.50(\mathrm{~m}, 1 \mathrm{H}), 2.59-2.65(\mathrm{~m}, 1 \mathrm{H}), 2.76-2.81(\mathrm{~m}, 1 \mathrm{H})$, 4.77 (brs, 1H), 5.54 (dd, $J=10.3,2.0 \mathrm{~Hz}, 1 \mathrm{H}), 6.33(\mathrm{dd}, J=17.1,2.0 \mathrm{~Hz}, 1 \mathrm{H}), 6.54(\mathrm{dd}, J=17.1,10.3$ $\mathrm{Hz}, 1 \mathrm{H}), 7.06(\mathrm{~d}, J=7.3 \mathrm{~Hz}, 1 \mathrm{H}), 7.16-7.23(\mathrm{~m}, 2 \mathrm{H}), 7.61(\mathrm{~d}, J=7.3 \mathrm{~Hz}, 1 \mathrm{H})$; HREI-MS calcd for $\mathrm{C}_{16} \mathrm{H}_{20} \mathrm{O}_{2}$ MW 244.1463, found $m / z$ 244.1473 $\left(\mathrm{M}^{+}\right)$; Anal. Calcd for $\mathrm{C}_{16} \mathrm{H}_{20} \mathrm{O}_{2}$ : C, 78.65; $\mathrm{H}, 8.25$. Found: C, 78.60; H, 7.95.

General Procedure for Preparation of 1-Hydroxy-2,2-dialkyl-1-propanoylindan Derivatives 6a-c. A mixture of 1-hydroxy-2,2-dimethyl-1-propenoylindan (2a) (74.6 mg, $0.344 \mathrm{mmol})$ and 10\% Pd/C (25 
$\mathrm{mg})$ in AcOEt $(10 \mathrm{~mL})$ was stirred under hydrogen atmosphere at $1 \mathrm{~atm}$ for $18.5 \mathrm{~h}$. The solution was filtered and the filtrate was evaporated in vасио. The residue was purified by column chromatography on silica gel with $n$-hexane-AcOEt $(20: 1)$ to give 1-hydroxy-2,2-dimethyl-1-propanoylindan (6a) (58.7 $\mathrm{mg}, 79 \%$ ) as a colorless oil.

1-Hydroxy-2,2-dimethyl-1-propanoylindan (6a). colorless oil; IR (neat) 3454, $1697 \mathrm{~cm}^{-1}$; ${ }^{1} \mathrm{H}$ NMR $\left(400 \mathrm{MHz} \mathrm{CDCl}_{3}\right) \quad 0.94(\mathrm{t}, J=7.3 \mathrm{~Hz}, 3 \mathrm{H}), 1.03(\mathrm{~s}, 3 \mathrm{H}), 1.08(\mathrm{~s}, 3 \mathrm{H}), 2.04(\mathrm{dq}, J=18.1,7.3 \mathrm{~Hz}, 1 \mathrm{H})$, $2.31(\mathrm{dq}, J=18.1,7.3 \mathrm{~Hz}, 1 \mathrm{H}), 2.89(\mathrm{~d}, J=16.0 \mathrm{~Hz}, 1 \mathrm{H}), 3.07(\mathrm{~d}, J=16.0 \mathrm{~Hz}, 1 \mathrm{H}), 4.54(\mathrm{~s}, 1 \mathrm{H}), 7.14$ $(\mathrm{d}, J=7.3 \mathrm{~Hz}, 1 \mathrm{H}), 7.20-7.31(\mathrm{~m}, 3 \mathrm{H})$; HREI-MS calcd for $\mathrm{C}_{14} \mathrm{H}_{18} \mathrm{O}_{2} \mathrm{MW} 218.1307$, found $\mathrm{m} / \mathrm{z}$ 218.1314 (M $\left.\mathrm{M}^{+}\right)$; Anal. Calcd for $\mathrm{C}_{14} \mathrm{H}_{18} \mathrm{O}_{2}$ : C, 77.03; H, 8.31. Found: C, 76.93; H, 8.21.

2,2-Diethyl-1-hydroxy-1-propanoylindan (6b). colorless oil; IR (neat) $3450,1698 \mathrm{~cm}^{-1}$; ${ }^{1} \mathrm{H}$ NMR (400 $\left.\mathrm{MHz} \mathrm{CDCl}_{3}\right) \delta 0.73(\mathrm{t}, J=7.4 \mathrm{~Hz}, 3 \mathrm{H}), 0.86(\mathrm{t}, J=7.4 \mathrm{~Hz}, 3 \mathrm{H}), 0.93(\mathrm{t}, J=7.2 \mathrm{~Hz}, 3 \mathrm{H}), 1.36-1.56(\mathrm{~m}$, $3 \mathrm{H}), 1.80$ (sext, $J=7.3 \mathrm{~Hz}, 1 \mathrm{H}), 1.94-2.04(\mathrm{~m}, 1 \mathrm{H}), 2.29-2.39(\mathrm{~m}, 1 \mathrm{H}), 2.88(\mathrm{~d}, J=16.4 \mathrm{~Hz}, 1 \mathrm{H}), 3.03$ $(\mathrm{d}, J=16.4 \mathrm{~Hz}, 1 \mathrm{H}), 4.57(\mathrm{~s}, 1 \mathrm{H}), 7.08(\mathrm{~d}, J=7.3 \mathrm{~Hz}, 1 \mathrm{H}), 7.18-7.28(\mathrm{~m}, 3 \mathrm{H})$; HREI-MS calcd for $\mathrm{C}_{16} \mathrm{H}_{22} \mathrm{O}_{2}$ MW 246.1620, found $m / z 246.1620\left(\mathrm{M}^{+}\right)$; Anal. Calcd for $\mathrm{C}_{16} \mathrm{H}_{22} \mathrm{O}_{2}$ : C, 78.01; $\mathrm{H}, 9.00$. Found: C, 77.80; H, 9.02.

2,2-Dibutyl-1-hydroxy-1-propanoylindan (6c). colorless oil; IR (neat) $3451,1698 \mathrm{~cm}^{-1}$; ${ }^{1} \mathrm{H}$ NMR (400 $\left.\mathrm{MHz}, \mathrm{CDCl}_{3}\right) \delta$ 0.80-1.80 (m, 21H), 1.90-2.05 (m, 1H), 2.28-2.41 (m, 1H), $2.88(\mathrm{~d}, J=16.3 \mathrm{~Hz}, 1 \mathrm{H})$, $3.05(\mathrm{~d}, J=16.3 \mathrm{~Hz}, 1 \mathrm{H}), 4.57(\mathrm{~s}, 1 \mathrm{H}), 7.07(\mathrm{~d}, J=7.3 \mathrm{~Hz}, 1 \mathrm{H}), 7.20-7.26(\mathrm{~m}, 3 \mathrm{H})$; HREI-MS calcd for $\mathrm{C}_{20} \mathrm{H}_{30} \mathrm{O}_{2}$ MW 302.2246, found $\mathrm{m} / z 302.2232\left(\mathrm{M}^{+}\right)$; Anal. Calcd for $\mathrm{C}_{20} \mathrm{H}_{30} \mathrm{O}_{2}$ : C, 79.42; $\mathrm{H}, 10.00$. Found: C, 79.56; H, 10.02 .

17ß-Hydroxy-3-methoxy-17 $\alpha$-propanoylestra-1,3,5(10)-trien (14). To a solution of methoxyallene $(421.9 \mathrm{mg}, 6.02 \mathrm{mmol})$ in dry THF $(6.0 \mathrm{~mL})$ was added $1.56 \mathrm{M} n$-butyllithium in $n$-hexane $(3.85 \mathrm{~mL}$, $6.0 \mathrm{mmol})$ at $-78{ }^{\circ} \mathrm{C}$ under argon atmosphere, and the mixture was stirred at $-78^{\circ} \mathrm{C}$ for $30 \mathrm{~min}$. Then the mixture was added dropwise to a dry THF $(40.0 \mathrm{~mL})$ solution of 3-methoxy-estra-1,3,5(10)-trien-17-one (8) $(568.8 \mathrm{mg}, 2.0 \mathrm{mmol})$ at $-78{ }^{\circ} \mathrm{C}$ in a cannula manner. After being stirred at $-78{ }^{\circ} \mathrm{C}$ for $2 \mathrm{~h}$, the reaction mixture was acidified by $1 \mathrm{~N} \mathrm{HCl}$, homogenized with $\mathrm{MeOH}$, and then the temperature was raised to room temperature. After being stirred for $1.5 \mathrm{~h}$, the reaction mixture was extracted with ether, and the ether extract was washed with brine, dried over $\mathrm{MgSO}_{4}$, and filtered. The filtrate was evaporated in vacuo to afford a crude product 9 . A mixture of crude compound 9 and $10 \% \mathrm{Pd} / \mathrm{C}(228.0 \mathrm{mg})$ in $\mathrm{THF}$ $(20 \mathrm{~mL})$ was subjected to hydrogenation under $\mathrm{H}_{2}$ at $1 \mathrm{~atm}$ for $18 \mathrm{~h}$. The solution was filtered and the filtrate was evaporated in vacuo. The residue was purified by column chromatography on silica gel with $n$-hexane-AcOEt (5:1) to give $17 \beta$-hydroxy-3-methoxy-17 $\alpha$-propanoylestra-1,3,5(10)-trien (14) (549.9 $\mathrm{mg}, 80 \%)$ as colorless prisms.; $\mathrm{mp} 117-118{ }^{\circ} \mathrm{C}\left(\mathrm{CHCl}_{3}-n\right.$-hexane-Et $\left.2 \mathrm{O}\right)$; IR (KBr) 3536, $1697 \mathrm{~cm}^{-1} ;{ }^{1} \mathrm{H}$ NMR $\left(400 \mathrm{MHz} \mathrm{CDCl}_{3}\right) \delta 0.96(\mathrm{~s}, 3 \mathrm{H}), 1.02-1.10(\mathrm{~m}, 4 \mathrm{H}), 1.35-1.55(\mathrm{~m}, 4 \mathrm{H}), 1.61-1.68(\mathrm{~m}, 1 \mathrm{H}), 1.70-$ $1.77(\mathrm{~m}, 2 \mathrm{H}), 1.81-1.93(\mathrm{~m}, 2 \mathrm{H}), 2.10-2.17(\mathrm{~m}, 1 \mathrm{H}), 2.23-2.30(\mathrm{~m}, 1 \mathrm{H}), 2.32-2.40(\mathrm{~m}, 1 \mathrm{H}), 2.43(\mathrm{~s}, 1 \mathrm{H})$, 2.62-2.73 (m, 2H), 2.84-2.87 (m, 2H), $3.77(\mathrm{~s}, 3 \mathrm{H}), 6.63(\mathrm{~d}, J=2.7 \mathrm{~Hz}, 1 \mathrm{H}), 6.70$ (dd, $J=8.5,2.7 \mathrm{~Hz}$, $1 \mathrm{H}), 7.15(\mathrm{~d}, J=8.5 \mathrm{~Hz}, 1 \mathrm{H})$; HREI-MS calcd for $\mathrm{C}_{22} \mathrm{H}_{30} \mathrm{O}_{3} \mathrm{MW} 342.2195$, found $m / z 342.2165\left(\mathrm{M}^{+}\right)$; Anal. Calcd for $\mathrm{C}_{22} \mathrm{H}_{30} \mathrm{O}_{3}$ : C, 77.16; H, 8.83. Found: C, 76.91; H, 9.03. 